\title{
DE MODERNIDADES Y ALIMENTACIÓN: COMER HOY EN ESPANA
}

\author{
Mabel Gracia Arnaiz \\ Universitat Rovira i Virgili-España
}

Resumen: Las nuevas maneras de comer permiten constatar en España una cierta simplificación e individualización de las comidas y un aumento del número de ingestas diarias. Aunque estos cambios avalarían, en parte, la tesis de la desestructuración propuesta para definir la modernidad alimentaria, nuestra hipótesis plantea que los comportamientos de los españoles se han modificado y diversificado adaptándose a los nuevos constreñimientos socioeconómicos impuestos por una sociedad cada vez más industrializada. En este sentido, las prácticas observadas no constituyen, salvo excepciones, síntomas alarmantes de una desestructuración generalizada, sino más bien, modos de comer más operativos y flexibles.

Palabras clave: cambios socioeconómicos, desestructuración alimentaria, flexibilidad, maneras de comer.

Abstract: The new eating manners state that, in Spain, there are some simplifications and individualization of meals and an increase in the number of daily intakes. Although these changes would partially support the thesis of the non-structural meals proposed to define the food modernity, our hypothesis suggests that the Spanish eating behaviours have changed and diversified to adapt to the new socioeconomic constrictions imposed by an increasing industrialized society. The food practices observed didn't show any disturbing symptoms of a general dysfunction but instead it showed the eating manners as more operational and flexible.

Keywords: eating manners, flexibity, non-structural meals, socioeconomic changes.

Numerosos estudios sobre las maneras de comer en las sociedades industrializadas han tenido como finalidad analizar hacia donde va la alimentación, tomando como punto de partida la evolución del consumo alimentario y las 
actitudes de los distintos grupos sociales ante las nuevas ofertas de alimentos. No obstante, la mayoría de estos estudios han privilegiado una orientación económica (interesándose por las compras y la evolución del gasto) o nutricional (interesándose por la evolución de la dieta). Desde estas perspectivas, las prácticas actuales, incluidas bajo el paraguas de la denominada modernidad alimentaria, son objeto de inquietudes facultativas y de incertidumbres socio-económicas sobre las que se debe actuar e intervenir, en cuanto que "las personas de las sociedades modernas no saben comer". Algunas de las hipótesis formuladas desde la antropología y la sociología aceptando la desestructuración de las maneras de comer contemporáneas han sido utilizadas para reafirmar, en buena medida, tanto la intervención médico-nutricional como el negocio de la industria alimentaria, mientras que otras hipótesis han criticado abiertamente la aceptación de esta supuesta degradación. En el centro del debate está la necesidad de diseñar estudios que metodológicamente puedan caracterizar las maneras de comer y dar cuenta de los factores que verdaderamente determinan las prácticas alimentarias, su transformación o su inmutabilidad, en tanto que sólo así se podrán explicar la naturaleza de dichas tendencias y la lógica que subyace en las elecciones alimentarias contemporáneas.

Las actuales maneras de comer en las sociedades industrializadas presentan una cierta ambigüedad respecto a su caracterización. ${ }^{1}$ Si bien la constatación de una cierta simplificación e individualización de las comidas o el aumento del número de ingestas diarias avalarían parcialmente la tesis de la desestructuración propuesta por Douglas (1979), Fischler (1979) o Herpin y Verger (1991) para definir la modernidad alimentaria, nuestra hipótesis plantea que aunque los comportamientos alimentarios de los españoles se han modificado y diversificado adaptándose a los nuevos constreñimientos socioeconómicos impuestos por una sociedad cada vez más industrializada, las

\footnotetext{
1 En este artículo se presentan los resultados de un estudio realizado con el soporte del Ministerio de Educación y Ciencia, La alimentación contemporánea desde y más allá de las normas (SEJ200615526-C02 02/SOCI). Los objetivos principales consistieron en conocer los factores y circunstancias que orientan y determinan las nuevas maneras de comer de los españoles, caracterizarlas y finalmente demostrar las diferencias existentes entre las normas y las prácticas alimentarias. Para ello, se organizó el trabajo de campo dos fases complementarias. La primera, de base etnográfica, se realizó mediante grupos de discusión y entrevistas en profundidad y la segunda, de base estadística, consistió en aplicar un cuestionario individual a una muestra representativa de la población española. Una presentación resumida de los resultados ha sido publicada en Gracia Arnaiz y Contreras Hernández (2006) y en Gracia Arnaiz (2008).
} 
prácticas observadas no constituyen, salvo excepciones síntomas alarmantes de una desestructuración generalizada, sino más bien, asumiendo la propuesta de Warde (1997), Bearsdworth y Keil (1997), Poulain (2002) o Ascher (2005) modos de comer más operativos y flexibles.

\section{Cambios sociales, cambios alimentarios}

Cuando hablamos de comidas nos estamos refiriendo a un conjunto diverso y complejo de prácticas relacionadas con el consumo de los alimentos: el perfil de las jornadas alimentarias, los comensales, los horarios y los lugares de las comidas, los tipos de menús, sus estructuras y contenidos, etc. Y, en tanto que la alimentación constituye una parte de nuestra realidad social y, al mismo tiempo, un reflejo de la misma, cabe considerar que los procesos de cambio experimentados por la sociedad española en los últimos treinta años han incidido, y se reflejan, en las actitudes y los valores de los actores sociales ante la comida, así como en los consumos alimentarios y en las particulares formas que éstos adoptan.

Podríamos decir que, si bien la alimentación ha sido siempre un hecho muy complejo y multidimensional, en nuestra modernidad lo es todavía más como consecuencia de la mayor diversidad sociocultural que hoy está presente en múltiples aspectos de la cotidianidad. Así, las comidas se han diversificado considerablemente en diferentes aspectos dando lugar a innovaciones, adaptaciones, sustituciones y desapariciones de productos, de recetas o prácticas como consecuencia, entre otros muchos factores, de

- las disponibilidades económicas de acuerdo con un presupuesto familiar en el que otros ítems de consumo han adquirido, comparativamente, una importancia mucho mayor (higiene, educación, transporte y ocio, por ejemplo);

- la mayor oferta alimentaria y su inclusión de los llamados alimentosservicio o de conveniencia;

- los horarios laborales y de otras actividades que pueden desarrollarse a lo largo de los diferentes días de la semana;

- la necesidad de compatibilizar la diversidad de horarios de los diferentes componentes de cada hogar; 
- las percepciones de los requerimientos alimentarios y/o nutricionales de cada uno y del grado de interés/voluntad por cumplirlos;

- el diferente grado de vinculación o apego de cada persona y/o grupo familiar respecto al sistema hegemónico de reglas sociales;

- la adaptación de las comidas a partir de las preferencias y las competencias culinarias personales tanto en lo relativos a alimentos como a prácticas alimentarias;

- las diversas concepciones sobre las relaciones entre salud, comensalidad, placer y sociabilidad y las diferentes formas y grados de satisfacer sus objetivos;

- etc.

La combinación de estos factores pueden explicar, al menos en parte, la falta de correspondencia que se manifiesta en el hecho de que, durante las últimas tres décadas, se hay producido, a pesar de que la salud constituye una preocupación básica, un descenso significativo del consumo de los alimentos considerados más "saludables" (verduras, hortalizas, legumbres) en favor de otros alimentos considerados menos "saludables" (carnes, pastelería industrial, snacks, etc.). Y es que la salud, aún siendo muy valorada por la mayoría de los españoles, no es la única ni la más determinante razón que guía las elecciones alimentarias: "Yo no compro algo que no le guste a mi familia, por muy sano que sea."

En este trabajo hemos estudiado cómo categorías tales como alimentos "buenos/malos", "adecuados/inadecuados" o "cómodos/incómodos", comer bien, comer bueno o comer sano... pueden tener significados muy diferentes, más o menos complementarios o contradictorios, si lo que está en juego es la salud, la hospitalidad o diferentes tipos de conveniencia o pragmatismo. Por ejemplo, la compatibilidad entre comer bien y comer sano, o comer bueno y comer sano, no siempre resulta fácil. Si "comer bien" se asocia casi indefectiblemente con "comer sano", "comer bueno" es entendido de manera muy diferente. No deja de ser significativo que, en materia alimentaria, dos términos tan próximos entre sí como son "bien" y "bueno" se entiendan de manera tan diferente. Así, por ejemplo, "bueno", cuando se habla de comer, se asocia con "rico" más que con "sano", es decir, más con el gusto que con la salud. "Comer bueno quiere decir comer buena calidad, lo que a ti te gusta, depende de lo que tu quieras": 
Para mí, comer bien, aparte de que la comida tenga una calidad, es no abusar de las grasas y comer, en general, de todo, llevar una dieta que decimos mediterránea: comer verduras, pasta, sobre todo mucha fruta y, indudablemente, también comer lentejas o cualquier cosa de éstas normalmente, y plancha, mucha plancha.

Comer bien quiere decir comer productos de buena calidad, lo que te gusta, lo que quieres.

Comer bueno significa comer lo que le gusta a uno. Comer con gusto. El tiempo es importante para comer con placer.

\section{Las comidas [1]: Comensalidad, lugares y horarios}

De todas las tomas alimentarias realizadas durante la jornada, la comida del mediodía y la cena son las que, hoy por hoy, tienen una mayor dimensión social. La importancia concedida al hecho de comer solo o en compañía no es la misma para cada tipo de comidas y la importancia tampoco es la misma si la comida se efectúa en casa o fuera de casa. Dicha comensalidad varía, también, dependiendo del tipo de comida y del momento en que se realiza. Así, por ejemplo, las comidas festivas, en contraposición con las comidas de los días ordinarios, se caracterizan por un mayor número de comensales y por una estructura y preparación más elaborada:

A mí me encanta cocinar, pero no para dos personas, sino para siete u ocho. $\mathrm{Y}$ sentarme y ver que están todos comiendo. A mí me chifla. Sí porque yo, de lunes a viernes, nunca cocino y, entonces, el sábado, un día que puedo, me apetece meterme en la cocina y hacer un buen asado o una paella.

Yo, siempre que ceno fuera de casa, es porque ceno para gozar de la cena y de la compañía y cuando me la hago en casa, rara vez lo hago también para lo mismo.

Durante la semana, el porcentaje de comidas que se realizan en compañía oscilan entre el $63,3 \%$ y el $65,2 \%$, siendo las menos compartidas el desayuno y las "pequeñas comidas" hechas entre horas (almuerzo, merienda y pica-pica). El fin de semana, en cambio, este porcentaje se incrementa, situándose en el $70 \%$ el sábado y el 69,8\% en domingo. Aunque leves, las diferencias permiten 
afirmar que durante los fines de semana se come más en compañía que los días laborables. En general, la comensalidad continúa presentando un fuerte protagonismo en las comidas: en 2 cada 3 ocasiones, los españoles comen acompañados $(65,7 \%)$. En los días laborables, los comensales proceden del ámbito de trabajo, de formación u ocio en detrimento de la compañía familiar. Por otro lado, comer en casa no significa necesariamente comer acompañado: esto queda ilustrado perfectamente en los desayuno e, incluso, las cenas.

En efecto, si bien la cena aparece como una ingesta mayoritariamente realizada en el ámbito doméstico, no necesariamente tiene el carácter familiar de antaño. Se produce, en este sentido, una desincronización los empleos del tiempo están cada vez menos coordinados de cara a hacer de la comida una actividad común:

¿La cena? ¡Cada uno viene a su hora!... Por la noche la cocina de casa funciona sin parar. Cada uno come cuando llega y coge lo que hay y si ya no queda nada, como son mayores, se preparan lo que les apetece.

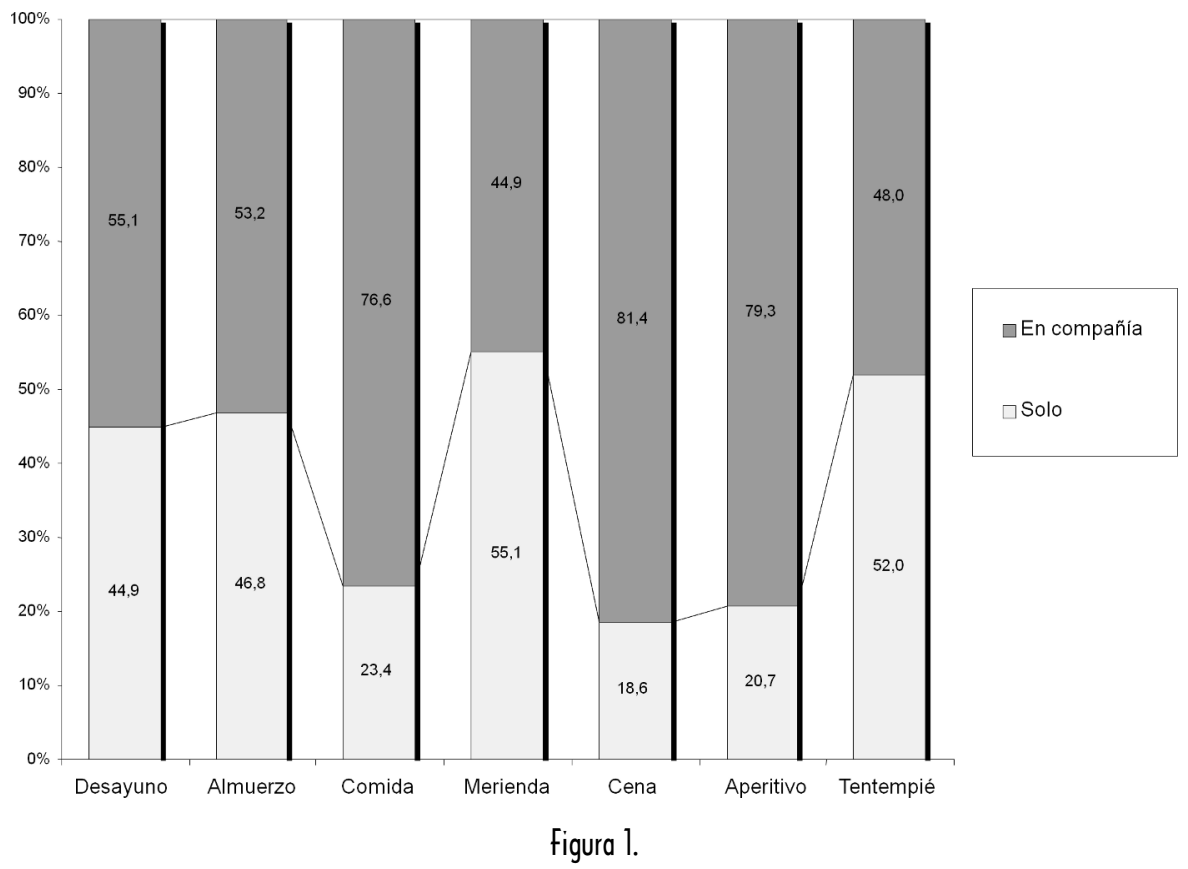

Horizontes Antropológicos, Porto Alegre, ano 16, n. 33, p. 177-196, jan.jun. 2010 
También se destaca que, aunque prevalece la idea de que cada vez se come con mayor frecuencia fuera de casa, el hogar continúa siendo el lugar donde se realizan la mayor parte de las comidas del desayuno, de mediodía y las cenas $(90 \%, 88,3 \%$ y $90 \%$ respectivamente). Contrariamente a lo que cabía esperar no se ha registrado un aumento importante en número de ingestas hechas fuera de casa, especialmente en la comida del mediodía. Sólo un $11,8 \%$ de las comidas del mediodía son realizadas fuera de casa, recurriendo para ello a la restauración privada, a comedores empresariales o escolares.

Relacionado con estos cambios, se está produciendo un fenómeno interesante relativo a la introducción de la alimentación en el lugar de trabajo que muestra, también, la naturaleza de la modernidad alimentaria. En efecto, está aumentando la realización de la comida del mediodía no ya en los restaurantes o cantinas de las empresas o escuelas, sino en el mismo despacho o en las salas de reunión y descanso mediante el recurso de la fiambrera: una comida elaborada a menudo en casa para ser consumida en los centros de trabajo o estudio. Las razones apuntadas para llevarse al trabajo la comida preparada en casa coinciden, parcialmente, con las dadas para comer fuera de casa (la falta de tiempo, la distancia entre el hogar y el lugar de trabajo y estudio) y se añaden otras más específicas como, por ejemplo, el ahorro económico, el mantenimiento de una cierta dieta e, incluso, el hecho de poder comer "más a gusto". Los alimentos se traen del propio domicilio o son comprados cerca del lugar de trabajo. Las motivaciones de estas prácticas, que afectan sobre todo a las mujeres cuadro y a los empleados, no son de tipo económico ni tienen que ver con un eventual descontento con el restaurante de la empresa sino con una lógica de organización del tiempo de trabajo [o del tiempo en general]. Razones esgrimidas a favor de la "fiambrera" o del "taper" tienen que ver con las oportunidades de hacer una comida más rápida $\mathrm{y}$, al mismo tiempo, considerada más sana o de poder realizar alguna otra actividad poco compatible con los horarios laborales sino se recurriera a la fiambrera:

Mi hija, ahora, tiene dos horas para comer al mediodía. Y, ahora, se van a la piscina. Se van todas las compañeras, se llevan su taper hecho de casa, sí. Es que, en verano, además cambia muchísimo la comida, porque normalmente coges una ensalada, una ensaladilla, cualquier cosa fría y en un momento te lo tomas, no es lo mismo que tener que calentártelo.

Yo prefiero llevarme algo de casa, y ligero, porque como te metas un plato o dos platos, el postre o no sé qué, llegas a la oficina y estás así de hinchada. 
Paralelamente, se han modificado los lugares de la casa donde se realizan las comidas y también las que se realizan en el exterior: "“Comer en el sofá? ¡Jamás! Bueno, sí, sábados y domingos por la noche.....”']: la "comida tradicional" tenía lugar en la cocina o en el comedor. Las "nuevas formas de comer", confirmando una desocialización parcial de las comidas, no están tan precisamente localizadas dentro de los diferentes espacios del hogar ni tampoco en el exterior. En el hogar, a los tradicionales espacios de la cocina y el comedor, se añaden la habitación ( $0,9 \%$ de las meriendas y $0,5 \%$ de las cenas) y el sofá (10,6\% de las cenas). En el exterior, al restaurante, el café y el comedor de empresa, se añaden la calle y los parques o jardines $(2,1 \%$ de las comidas del mediodía) y espacios más o menos indeterminados dentro de los lugares de trabajo.

Yo prefiero comer en casa, sin duda. Pero no lo hago ningún día, o sea, no puedo. Es una cuestión de horarios. Como fuera siempre.

A veces (sus hijos) meriendan en el parque o por la calle, depende, si después tienen que ir a música o natación...

Respecto a los horarios, el incremento de la dispersión es notable. ["Nosotros somos cuatro y ;comemos cada uno a una hora diferente!"]: las "nuevas" comidas no tienen lugar a horarios fijos. Ni el principio ni el final de las diversas comidas se sitúan dentro de franjas horarias estrechas. Esta imprecisión de las fronteras horarias hace que aparezcan formas híbridas como la "merienda-cena" o el "aperitivo-comida". Es cierto, no obstante, que cada comida tiene unas franjas horarias y un punto modal en que se realizan un mayor número de ingestas. En el siguiente gráfico sólo se han representado las cinco comidas principales (desayuno, almuerzo, comida, merienda, cena). Como se observa con claridad, cada una de las cinco comidas tiene su franja horaria propia, si bien se solapan parcialmente entre ellas. Así, el desayuno se suele realizar entre las 4 y las 13 horas, situándose el punto máximo a las 8 horas. El almuerzo o desayuno de media mañana, también las once, se realiza entre las 7 y las 16 horas, con un punto máximo a las 11 y un segundo pico a las 14 horas. La hora de la comida se sitúa entre las 11 y las 18 horas, con un punto máximo a las 14 horas. La merienda, a continuación, se realiza desde las 14 hasta las 23 horas, si bien su punto horario álgido son las 18 horas. Finalmente, la hora de la cena más habitual es a las 21 horas, si bien la franja horaria comienza a las 18 y se alarga hasta la madrugada (Figura 2). 


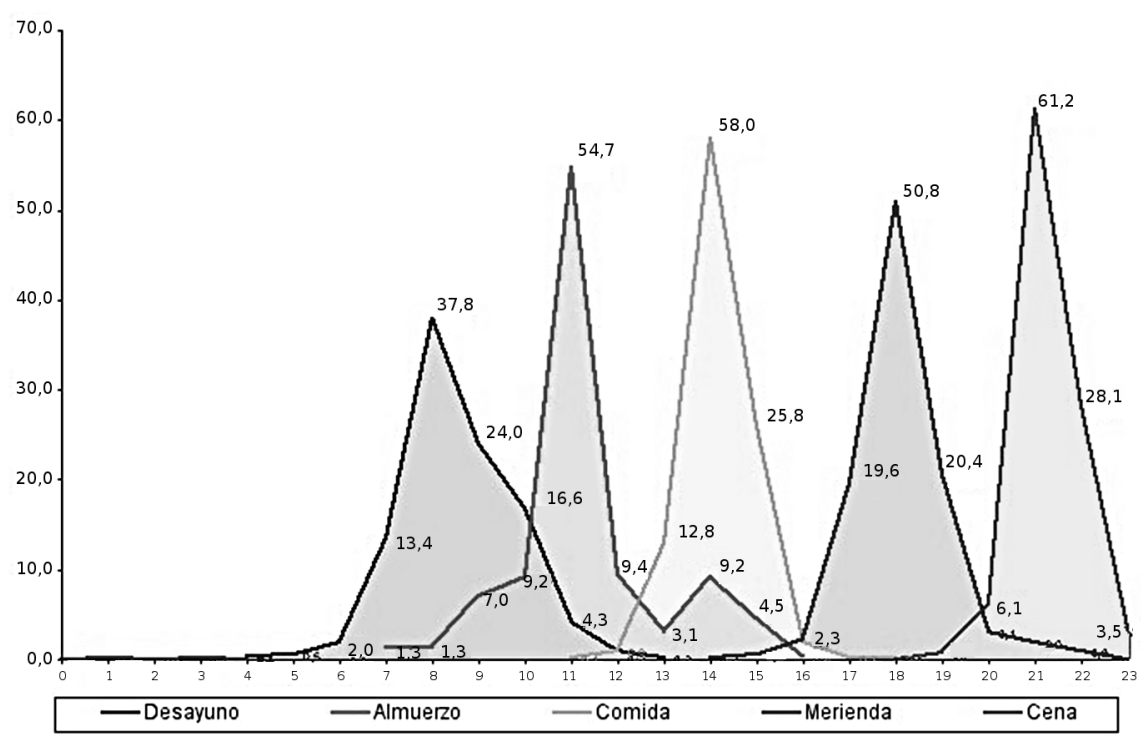

Figura 2 .

En una primera instancia, puede sorprender la amplitud horaria de las ingestas, esa base amplísima que muestra gráficamente que el desayuno, por ejemplo, pueda realizarse, según los casos, entre las 4 de la madrugada o a las 13 horas, pasado el mediodía. Sin embargo, es simplemente, la expresión de la enorme diversidad de situaciones y horarios existentes desde diferentes puntos de vista (laborales, formativas o de ocio). Así por ejemplo, la existencia de turnos de trabajo (horario de día o de noche) en muchas empresas y servicios que funcionan las 24 horas del día condicionan la alimentación y sus horarios por varias razones. Así, cuando se trabaja en el turno de noche, alguno puede no cenar o no comer ya que se levanta tarde al día siguiente. Como consecuencia de la diversidad de horarios laborales y de la existencia de turnos en las empresas, muchas personas no guardan un horario más o menos fijo para las diversas comidas.

Cada tres semanas suelo hacer una semana de noche. Trabajo en un hospital y, la semana que estás de noche, andas un poco despistado, al mediodía no comes 
porque te levantas tarde, el día que te vas a trabajar, no cenas, y luego te comes un sándwich a las cuatro de la mañana, o cosas así. No comes con la familia porque no te coincide.

Además, cabe tener en cuenta, también, la diversidad de horarios existente entre los días laborales por un lado y los fines de semana, los festivos y las vacaciones por otra. Los días festivos, días de ocio, se caracterizan, entre otros aspectos, por una evasión de las obligaciones y de las formalidades y, en ese sentido, los constreñimientos horarios, y entre ellos también los de las comidas, son uno de los aspectos de los que se quiere huir:

Normalmente, el domingo me levanto a las doce, a la una. Comes a las cuatro, si comes... porque has comido una tapas, luego en el cine unas palomitas y ya, por la noche no tienes hambre.

\section{Las comidas [II]: número de comidas diarias, estructuras y contenidos}

En relación con la jornada alimentaria, la población española declara seguir un modelo ternario (desayuno, comida y cena) y tripartito (primer plato, segundo plato y postres). Sin embargo, los resultados del estudio indican, respecto al número de ingestas realizadas, una media superior a las cuatro tomas diarias y, frente a las estructuras ternarias, un aumento progresivo de las binarias e, incluso, una reinstauración del denominado "plato único". La tendencia de las principales comidas se orienta, en consecuencia, en el sentido de la simplificación y la concentración.

La mayoría de los informantes afirman comer entre tres y cuatro veces al día (38\% y $38,5 \%$ respectivamente), mientras que el $21 \%$ dice hacer cinco ingestas. En el caso de realizarse tres, éstas hacen referencia al desayuno, la comida y la cena, añadiéndose en la mayoría de casos la merienda. Cuando se apuntan las cinco ingestas, éstas incluyen, además de las anteriores, el almuerzo de media mañana u once. El 2,5\% restante incluye aquellos que dicen comer dos veces al día -el $52 \%$ de esos casos- y entre seis y once veces diarias -el 48\%. En cualquier caso, vale la pena destacar que el modelo ternario (tres tomas diarias diferenciadas) está bastante interiorizado en cuanto son frecuentes las respuestas dadas en estos términos: 
Hago tres comidas fuertes y un aperitivo.

Tres comidas y dos picoteos.

Tres comidas y la merienda.

Desayuno, comida, cena y extras.

Normalmente tres, y no todos los días, algunos meriendo.

Tres, desayuno, almuerzo y cena, aunque suelo picar algo al mediodía.

Desayuno, comida y cena; a veces una pequeña merienda, poca cosa.

Tres, un poco a media mañana y a veces aperitivo.

Sin embargo, la descriptiva obtenida de las prácticas registradas indica que el número medio de ingestas diarias es ligeramente superior: se sitúa en 4,19, lo que supone una media total de 29,33 ingestas semanales. Si atendemos a los días de la semana de forma separada, el domingo disminuye el número de ingestas, situándose en 4,06, mientras el sábado mantiene un comportamiento similar al de la media total (Figura 3). Por ello, no se puede hablar tanto de una diferencia entre días laborables y fin de semana como entre domingo y el resto de los días.

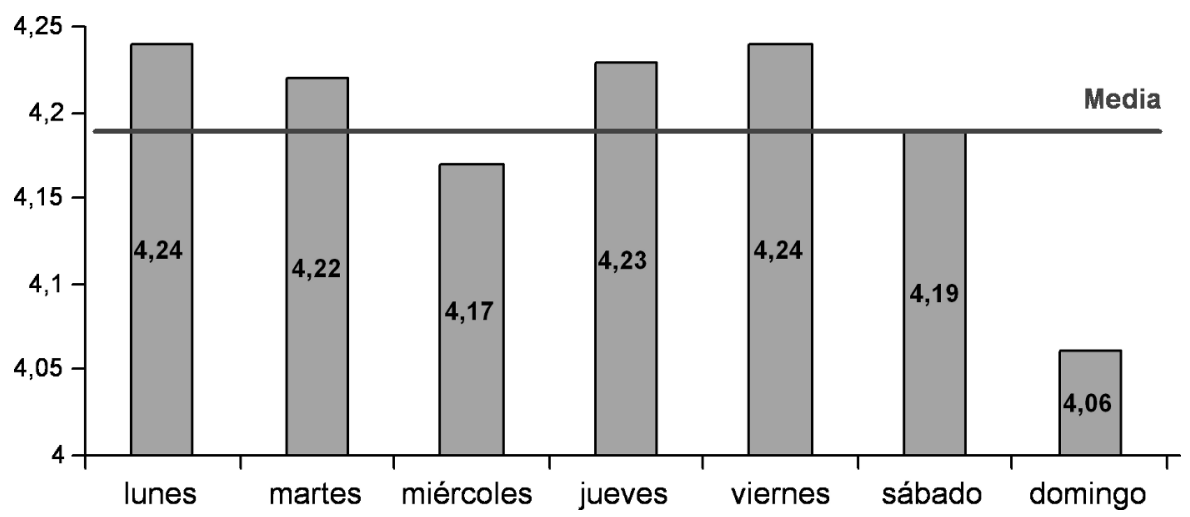

Figura 3. 
Conviene subrayar, en este sentido, que las "comidas" y los "alimentos" no se piensan ni se califican individualmente. Todas las personas son conscientes de que los alimentos suelen formar parte de "platos" y que las comidas están integradas en "ciclos", por lo menos de carácter semanal o incluso diario, de tal forma que la estructura y el contenido de una comida principal deben considerarse teniendo en cuenta los contenidos de las restantes. El caso del domingo es muy ilustrativo en tanto que, por ser día festivo, es la jornada más diferenciada del resto. Por un lado, un desayuno tomado a una hora más tardía de lo habitual contribuye a eliminar el almuerzo de media mañana y una comida más compleja, abundante, sustanciosa y tomada, también, a una hora más tardía de lo habitual en los días laborables contribuye a suprimir la merienda o tomas de media tarde o hacer una cena mucho más ligera.

Normalmente, el domingo me levanto a las doce, a la una. Comes a las cuatro, si comes... porque has comido unas tapas, luego en el cine unas palomitas y ya, por la noche no tienes hambre.

Se constata, así, que la alimentación ordinaria se alterna con acontecimientos más o menos extraordinarios (días festivos, vacacionales, celebraciones diversas, pensemos en las ingestas propias del ciclo navideños, por ejemplo) en los que los criterios que determinan lo que se come pertenecen a un orden muy distinto del de la nutrición, que marcan, a su vez, los diversos tipos de preferencias y, también, de incompatibilidades alimentarias.

Respecto a las comidas más habituales, el desayuno es la que abre la jornada alimentaria y consiste en una comida ligera, habitualmente dulce (bollería, galletas y dulces) acompañada de café y/o leche. Leche, cereales, tostada con aceite, fruta, cruda o en zumo... son, sin embargo, los ingredientes que se consideran ideales para un buen desayuno. Existe un acuerdo bastante generalizado al respecto. No obstante, cuando se entra en cuál es el desayuno real de cada persona, los matices y/o las excepciones son constantes:

Para mí, tiene que ser zumo, cereales, y fruta, pero...

Para mí sería lo ideal (leche, cereales, tostada con aceite, fruta...), que yo no lo hago porque no tengo tiempo.

Me gustaría desayunar fuerte para evitar el almuerzo, pero soy incapaz de madrugar y ponerme a desayunar una tostada, un huevo, fruta, no puedo... 
Así pues, si bien existe un consenso generalizado con relación a lo que se considera un "buen" desayuno, tanto en términos estrictamente energéticos como en términos de lo que es el desayuno adecuado en términos de salud, una mayoría de personas reconocen o expresan diferente tipo de problemas o condicionamientos que les impiden o dificultan llevarlo a la práctica. Así, la "falta de tiempo" o, simultáneamente, "la falta de tiempo y la pereza" son una limitación para poder cumplir con lo que se considerar debiera ser "un buen desayuno", de forma que la convierten en la ingesta más ligera de las tres comidas principales que casi obliga, especialmente entre aquellas personas que comen tarde más allá de las 14 o 15 horas, a realizar el almuerzo de media mañana.

De toda la jornada alimentaria, la comida del mediodía es la que tiene una mayor relevancia social y nutricional:

Platos de cuchara y, sobre todo, carne.

Un plato fuerte y ensalada; carne-patatas, pasta-carne, verduras-huevos.

Es la comida fuerte del día: carne y algo de pasta, con ensalada,

Platos que nutran, proteínas, legumbres, carnes.

También es la que presenta un mayor grado de estructuración, aunque el modelo ternario no sea, como se piensa, el más frecuente, ya que 1 de cada 2 comidas corresponden a estructuras binarias, es decir, compuestas por dos platos o elementos $(47,5 \%)$, mientras que las estructuras ternarias se sitúan en segundo lugar $(29,2 \%)$ y sólo el $0,4 \%$ son cuaternarias (Figura 4$)$.
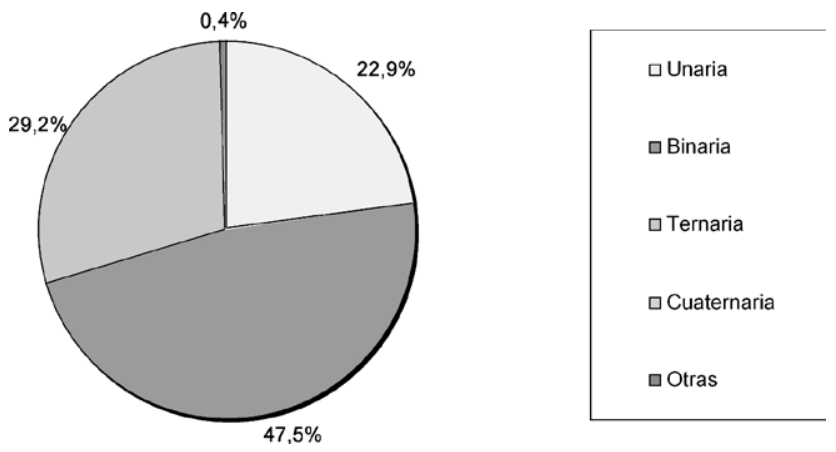

Figura 4. 
Respecto al contenido, la carne se consume en un 56,9\% de las comidas de los españoles, mientras la fruta se consume en un 50\%, siendo estos dos alimentos, claramente, los más característicos de este tipo de ingesta. A continuación, con una proporción bastante elevada se sitúan las verduras y hortalizas $(39,1 \%)$, y un poco por debajo la pasta y el arroz $(29,8 \%)$ y las ensaladas y gazpachos $(27,3 \%)$. El pescado $(21,2 \%)$, las patatas $(22,5 \%)$ y, algo menos, las legumbres también son alimentos habituales en las comidas de españolas (Figura 5). La bebida más habitual es el agua (56,7\%), seguida a cierta distancia del vino $(21,5 \%)$.

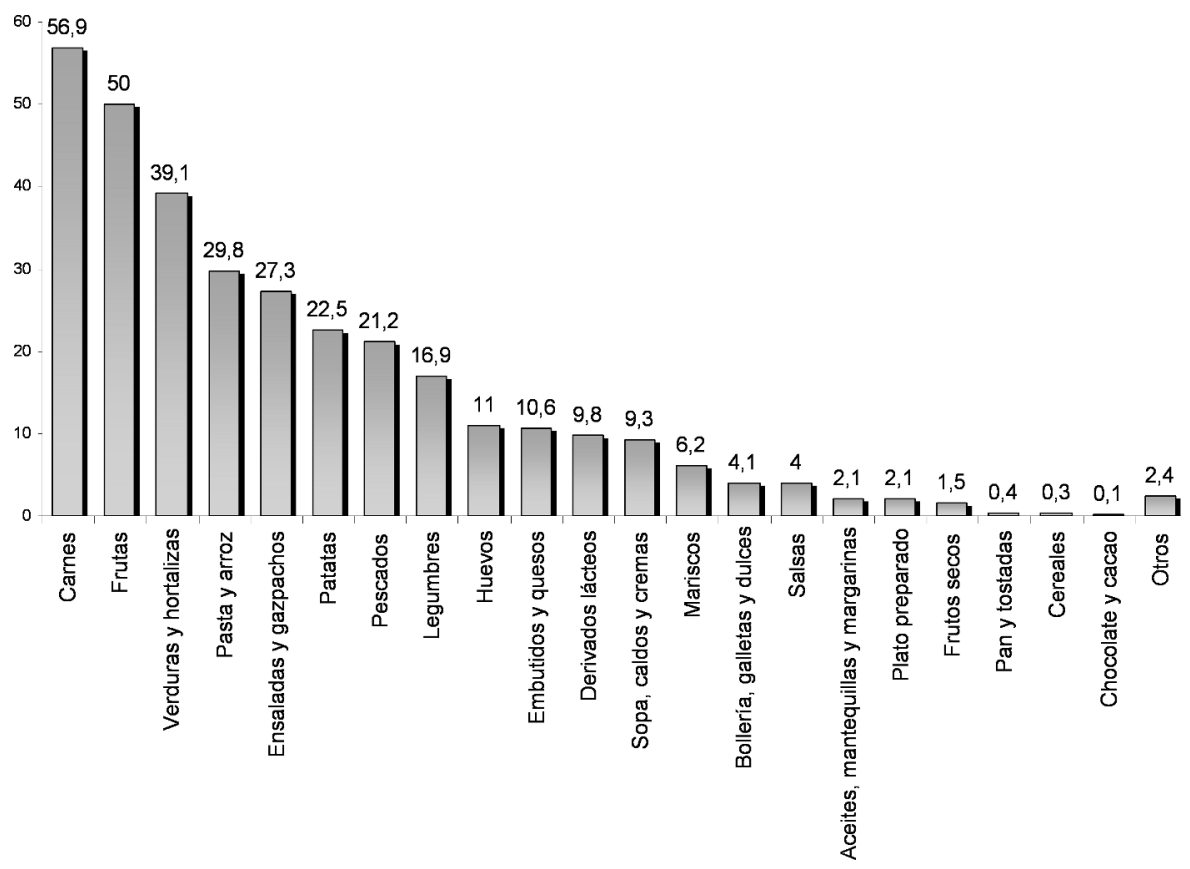

Figura 5 .

Del análisis de las combinaciones alimentarias hechas en esta comida se extraen observaciones interesantes. Por ejemplo, si nos centramos en el segundo plato, vemos que se están realizando nuevas conjugaciones: puede 
mantenerse un producto base o estructurante (por ejemplo, la carne o el pescado) pero cambiando lo que se consideraba tradicionalmente como guarnición. Siguen dominando las ensaladas y las patatas como acompañantes pero, en numerosas ocasiones, la variedad es tanta que puede que lo que fue un segundo plato se convierte en un plato combinado y, en esta misma medida, en plato único. En otros casos, el elemento base puede no ir acompañado de la guarnición.

Asimismo, se observa que los productos considerados centrales o de base y alrededor de los cuales acostumbraba a girar una gran variedad de productos empiezan a verse sustituidos por otras variedades alternativas. Por ejemplo: los productos cárnicos más habituales (pollo, bistec de ternera, cerdo) empiezan a ser reemplazados por otros alternativos como las salchichas "de Frankfurt", las hamburguesas, los "San Jacobo", etc. Así, se observa la "aparición" de platos tales como "san Jacobo con verdura", "hamburguesa con ensalada", etc. Subsisten, no obstante, los "tradicionales" platos únicos (como "paella", "lentejas", etc.) que, acompañados de un postre, componen íntegramente una comida principal.

En cuanto al postre, la fruta es el producto más frecuente, sobre todo en las comidas ordinarias o no festivas y en el propio domicilio. Los helados aparecen, también, con mucha frecuencia pero, sobre todo en las comidas fuera de casa o en los días festivos o fines de semana. Los zumos de fruta, también los yogures, se consideran ya un postre. Y, en muchos casos, el postre, simplemente, no aparece y es substituido por el café, lo que da lugar a una reducción parcial de lo que se considera la estructura tradicional de las comidas principales, que pasa de ternaria a binaria.

La cena, a diferencia de la comida del mediodía, no se entiende como la comida más importante del día, aunque si tiene el estatus de comida principal, formando parte de una de las ingestas que es incluida en el repertorio de la casi totalidad las personas entrevistadas. En las cenas se agudizan algunas de las tendencias acabadas de apuntar, sobre todo el fenómeno de la concentración o condensación de la comida en un solo plato (plato único o plato combinado). En general, la cena se ha convertido en la comida que mayor grado innovación registra en su estructura, habitualmente binario y cada vez más unaria, tanto en términos de ingredientes, como de sucesión y combinación de los alimentos. Una posible causa de estos cambios puede encontrarse en el hecho de que muchas personas, incluidos los más pequeños, resuelven la comida principal 
más estructurada y de elaboración más compleja del día fuera de casa, lo que legitima a la gente a solucionar las cenas domésticas con "cualquier cosa" y "de cualquier manera":

Lo que complemente lo que no he tomado en la comida.

Alimentos fáciles de preparar, pescado hervido, y algún producto lácteo o fruta.

Algo que te haga hacer bien la digestión: fruta, tortilla, puré, verdura.

Ni demasiado fuerte ni abundante.

En las cenas, en efecto, aparecen con mucha frecuencia platos en los que pueden figurar una serie de productos más o menos variados y, a su vez, difíciles de clasificar o agrupar, pero sobre todo, más rápidos de preparar: embutidos, fruta, ensaladas, bocadillos, platos preparados, platos étnicos (de

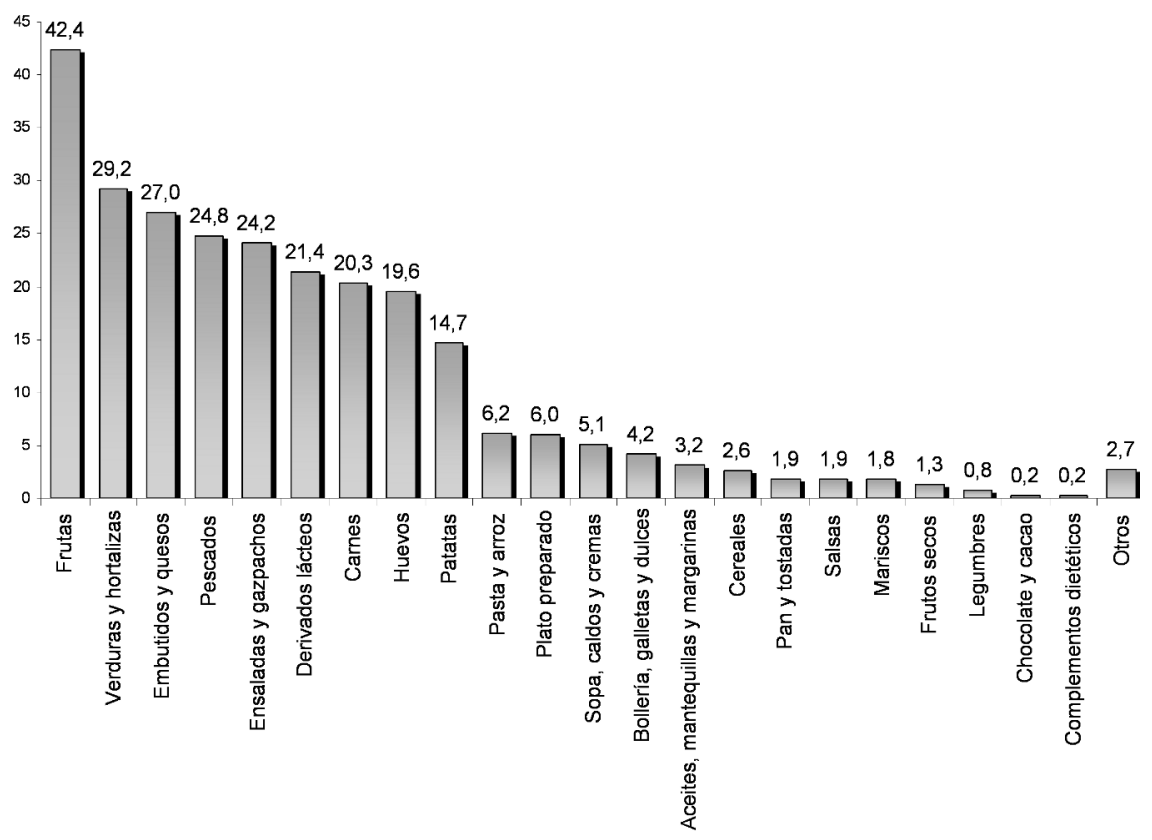

Figura 6.

Horizontes Antropológicos, Porto Alegre, ano 16, n. 33, p. 177-196, jan./jun. 2010 
origen chino, por ejemplo), derivados lácteos, etc. La fruta es, con diferencia, el alimento más habitual (42,4\%), seguido a cierta distancia de las verduras y hortalizas, de los quesos y embutidos y también del pescado y los derivados lácteos (Figura 6).

Por su parte, las "pequeñas comidas" o las no-comidas realizadas entre horas (almuerzo, merienda, tapeo, aperitivo, pica-pica), aun teniendo una presencia notable en los registros individuales, son consideradas por una gran mayoría de los adultos como evitables o, incluso, de forma negativa, es decir, como comidas que deberían suprimirse ya sea por motivos de salud y/o de estética. Ello no impide que más de 1/3 parte de la población españoles practique el snacking de forma habitual y que se haya producido una transferencia de los ingredientes de las comida principales (desayuno, comida y cena) en beneficio de las "pequeñas" comidas. Por ejemplo, el "bocata" o "tentempié" de la mañana o el "bocata" de media tarde que hace, en numerosas ocasiones, de merienda-cena:

No como grandes cantidades nunca, pero sí que las espacio pues estoy picando todo el día. Entonces, seguro que hay días que, a lo mejor, como tres veces; y otros días, siete, si se llama comer, comer un yogur o picar...

Lo que en realidad se ingiere para el "picoteo" o la comida "entre horas" es muy variado, dependiendo, principalmente, de la hora del día. Dentro de la categoría del pica-pica, en España hay que diferenciar entre el aperitivo y el tapeo. En efecto, otra modalidad de comer entre horas que recibe una denominación específica es "el aperitivo", ingesta que se antecede a la comida del mediodía. El aperitivo, por otro lado, está asociado con una comida principal que tenga un carácter excepcional, ya sea porque se trata de una ingesta de fin de semana ya sea porque antecede a una comida formal. El alimento más consumido por los españoles en los aperitivos son las patatas $(39,1 \%)$, concepto que en este caso debe ser entendido en sentido amplio, incluyendo tanto las propias patatas, como sus derivados (patatas chips y otros snacks asociados). En un $22,5 \%$ de los aperitivos se consume frutos secos.

Por su parte, tomar "tapas" tiene una percepción diferente que el "picar entre horas". Las tapas, siempre fuera del hogar, son percibidas como un tipo de comida preciso, anterior, por lo general, a la comida o a la cena y, en ocasiones, puede sustituir a una de esas comidas, sea por la abundancia de las 
tapas ingeridas o, precisamente, porque se pretende una comida más ligera. Las tapas, como antesala de una comida ordinaria, son más propias de los días festivos o de los fines de semana y de los períodos de vacaciones. "Ir de tapas" está muy relacionado con la sociabilidad y, a su vez, con una forma de comer considerada propiamente "española", aunque existen diferencias de unas regiones a otras. La gama de alimentos y de preparaciones susceptible de convertirse en tapas es casi ilimitada y, podría decirse que, a las tapas más o menos clásicas se les añaden nuevas tapas constantemente: boquerones en vinagre, magro con tomate, tortilla de patatas, callos con garbanzos...:

Como, normalmente, salgo mucho... Yo comprendo que no lo debería hacer pero... entre la cena y la cervecita, pues siempre guarreo... que si frutos secos, que si patatas fritas... Porque yo suelo salir bastante y eso es mi debilidad.

A mi el tapeo no me quita de comer... Me gusta sentarme el fin de semana, o sea, el sábado y el domingo, con mi mujer, y charlar comiendo, o sea se hace una comida en condiciones, lo que no hago en toda la semana.

Así pues, y como hemos podido observar en esta breve descripción del perfil de las jornadas alimentarias de los españoles, el repertorio de comidas no sólo es el resultado de las recomendaciones dietéticas, sino también de aquellas otras contra-indicaciones que se derivan de lo cotidiano y que son diferentes de aquellas que existían hace treinta años. Son los diferentes contextos quienes condicionan, en mayor o menor grado, las "decisiones" que son tomadas a nivel doméstico o a nivel individual, las cuales, a su vez, dependen de los diferentes tipos de objetivos (placer, comodidad, salud, sociabilidad, apetito, ritualidad, tradición, etc.) que pueden ser perseguidos en cada una de las diferentes ingestas porque, es necesario no olvidarlo, cada toma alimentaria, cada decisión alimentaria, no tiene por qué responder a una sola lógica y no todas las tomas tienen que responder siempre a la misma lógica o motivación. Lo dicho es obvio, pero no siempre se tiene en cuenta.

No es fácil prever la dirección de los cambios futuros. Los ciudadanos, animados por racionalidades complejas y a veces divergentes, efectúan elecciones alimentarias cotidianas cada vez más diversificadas, específicas e irregulares de tal modo que, en un contexto así, seguir una jornada alimentaria de una forma más o menos estructurada comporta una rutinización bastante 
constriñente. Esta es, probablemente, una de las razones que hacen que sea tan difícil seguir las recomendaciones nutricionales. La vida de la gente es más bien irregular, salpicada de numerosos micro-acontecimientos, cambios de programa de actividades, horarios variables, desplazamientos de todo tipo, que fragilizan el control que constituyen las rutinas dietéticas. En un contexto, además, en el que disminuye el tiempo dedicado a cocinar, se prefieren las técnicas de cocción más rápidas y aquellos alimentos que se prestan más a ello. Se trata de satisfacer varios y diversos objetivos: ahorrar tiempo en la preparación de los menús, así como en las tareas de poner o quitar la mesa; evitar al máximo los aspectos "sucios" del tratamiento de las materias primas y de limpieza post-cocina. Se trata, también, de gestionar de la manera más eficiente posible la diversidad de horarios, de necesidades y de preferencias de los diferentes integrantes del hogar. Opertatividad y flexibilidad es lo que demandan los nuevos estilos de vida a las maneras de comer contemporáneas. Estas circunstancias dejan un campo completamente abierto a la tecnología alimentaria y a sus aplicaciones industriales ya que, a lo largo de la historia, los conservadurismos alimentarios se han explicado por el hecho de que cada cultura ha transmitido en cada generación los principios culinarios básicos, los gustos propios y los valores dietéticos. Y hoy, las nuevas formas de vivir no garantizan, en buena medida, la continuidad de este proceso.

\section{Referencias}

ASCHER, F. Le mangeur hypermodern. Paris: Odile Jacob, 2005.

BEARSDWORTH, A.; KEIL, T: Sociology on the menu: an invitation to he study of food and society. London: Routledge, 1997.

DOUGLAS, M. Les estructures du culinaire. Communications, Paris, n. 31, p. $145-170,1979$.

FISCHLER, C. Gastro-nomie et gastro-anomie. Communications, Paris, n. 31, p. $189-217,1979$.

GRACIA ARNAIZ, M. Nuevas maneras de vivir, nuevas maneras de comer. Distribución y Consumo: Monográfico Sociología de la Alimentación, n. 97, Madrid, p. 5-17, enero/feb. 2008. 
GRACIAARNAIZ, M.; CONTRERAS HERNÁNDEZ, J. Manger aujourd'hui en Espagne: nouvelles tendances. Les Cahiers de l'OCHA, Paris, v. 1, n. 11, p. 106, 117, 2006.

HERPIN, N.; VERGER, D. La consommation des Français. Paris: La Découverte, 1991.

POULAIN, J-P. Manger aujourd'hui: attitudes, normes et pratiques. Toulouse: Privat, 2002.

WARDE, A. Consumption, food and taste. London: Sage, 1997.

Recebido em: 25/10/2009

Aprovado em: 26/03/2010 